\title{
The Effects of Thermoelectric Magnetohydrodynamics in Directional Solidification Under a Transverse Magnetic Field
}

\author{
A. Kao ${ }^{\mathrm{a}, *}$, B. Cai ${ }^{\mathrm{b}}$, P. D. Lee ${ }^{\mathrm{b}}$, K. Pericleous ${ }^{\mathrm{a}}$ \\ ${ }^{a}$ Centre for Numerical Modelling and Processes Analysis, University of Greenwich, Old \\ Royal Naval College, Park Row, London, SE10 9LS \\ ${ }^{b}$ The Manchester X-Ray Imaging Facility, School of Materials, The University of \\ Manchester, Oxford Rd., M13 9PL, UK
}

\begin{abstract}
The mechanism of macrosegregation and modification to dendrite size and spacing from a transverse magnetic field has been modelled through direct numerical simulation. The primary driver for this mechanism was identified as a strong Lorentz force formed in the interdendritic region, which leads to a large scale flow circulation. The microstructure evolution is modified by convective transport of solute and the predicted morphological features compare favourably to experimental data in the literature. The numerical results also give an insight into the magnitude of flow velocities within the interdendritic region.
\end{abstract}

Keywords: A1. Directional solidification, A1. Crystal structure, A1. Mass transfer, A1. Magnetic fields, A. Thermoelectric currents

\section{Introduction}

The application of an external magnetic field to a solidifying alloy has been experimentally observed to have a significant effect on microstructural evolution $[1,2,3,4,5,6,7,8,9,10,11]$. These changes can be attributed to convective transport of solute by Thermoelectric Magnetohydrodynamics (TEMHD). A detailed description of TEMHD is given by Shercliff [12], but in summary TEMHD describes fluid flow generated by a Lorentz force

*Corresponding author A. Kao, a.kao@gre.ac.uk, +44 2083317796 
that is formed through the interaction of thermoelectric currents and a magnetic field. Thermoelectric currents are generated by spatial variations in temperature and Seebeck Coefficient (absolute thermoelectric power). In directional solidification these conditions are satisfied by the externally driven thermal gradient and through ejection of solute at the liquid/solid interface respectively.

In the presence of a transverse magnetic field, macrosegregation on the sample scale has been observed for both in situ thin sample experiments [4] and post-mortem analysis of rod shaped samples $[4,5,6]$. Macrosegregation occurs normal to the magnetic field orientation and to the thermal gradient. Descriptions of the mechanism have been given by authors of these experimental findings $[4,6]$, supported with idealised steady state 2-dimensional models of TEMHD. They suggest that this behaviour is caused by interdendritic flow transporting high concentration of solute out of the mushy zone. However to date no, quantitative representation of the complete mechanism responsible for the observed macrosegregation, that couples between transport, solidification, electric current and flow has been presented in the literature. Numerical simulations of a similar system have been presented by [13], however the use of periodic boundaries could not account for the influence of the sample edges, thus any ejection of solute into the melt could not be captured. This paper investigates the underlying mechanism of TEMHD driven macrosegregation on a small sample through direct numerical simulation using a fully coupled, transient, 3-dimensional model that includes solidification, thermoelectric currents, electromagnetism and fluid flow [14].

\section{Governing equations}

Phase transition is tracked using an enthalpy conservation method based on the work of Voller [15]. The volumetric enthalpy $H$ is defined as the sum of sensible latent heats

$$
H=c_{p} T+f L
$$

where $c_{p}$ is the volumetric specific heat capacity, $T$ the temperature and $L$ the volumetric latent heat of fusion. The liquid fraction $f$ varies from $f=1$ when fully liquid to $f=0$ when fully solid; intermediate values $0<f<1$

represent solidifying cells. The interface equilibrium is given by the GibbsThompson condition

$$
T^{i}=T_{m}-\frac{\gamma(\theta, \phi)}{L} T_{m} \kappa-m\left(C_{0}-C_{l}^{i}\right)
$$


where $T^{i}$ is the interfacial temperature, $T_{m}$ the melting temperature, $\gamma(\theta, \phi)$ the surface energy anisotropy, $\theta$ and $\phi$ angles to the interface normal, $\kappa$ the curvature, $m$ the liquidus slope, $C_{0}$ the bulk solute concentration and $C_{l}^{i}$ the liquid solute concentration at the interface. The concept of a concentration potential $V$ is used to describe solute ejection in binary alloys and $V$ is defined as

$$
V=\frac{C}{f(1-k)+k}
$$

where $k$ is the partitioning coefficient. Transport of mass is given by

$$
\frac{d V}{d t}=\nabla \cdot D \nabla V-\mathbf{u} \cdot \nabla V
$$

where $t$ is time and $D$ is mass diffusivity. The Scheill assumption $(D=$ $0)$ is applied to the solid. Due to the low thermal Péclet number $(\mathrm{Pe}=$ $O 10^{-2}$ ) heat transport is assumed to be dominated by diffusion and the thermal diffusivity is assumed constant. Thus, in directional solidification the temperature field varies only in the direction of solidification and is known explicitly.

Thermoelectric currents can be described by a generalised form of Ohm's law

$$
\mathbf{j}=\sigma(\mathbf{E}+\mathbf{u} \times \mathbf{B}-S \nabla T)
$$

where $\mathbf{j}$ is the current density, $\sigma$ is the electrical conductivity, $\mathbf{E}$ is the electric field, $\mathbf{u}$ is the fluid velocity and $\mathbf{B}$ is the magnetic field. The final term is the thermoelectric field and $S$ is the Seebeck coefficient. In the presence of a magnetic field, the current interacts to give a Lorentz force $\mathbf{j} \times \mathbf{B}$, which drives flow in the liquid phase. Due to the relatively slow fluid velocity and the small length scales of microstructures it can be shown that the Reynolds number, Re $<1$. Thus incompressible Stokes flow can be assumed given by

$$
\rho \frac{d \mathbf{u}}{d t}=-\nabla p+\mu \nabla^{2} \mathbf{u}+\mathbf{j} \times \mathbf{B}
$$

where $\rho$ is the density, $p$ is pressure and $\mu$ is the viscosity.

The coupled equation set is solved using a bespoke code ThermoElectric Solidification Algorithm (TESA) developed by the authors. The code primarily consists of a set of weakly coupled finite difference schemes, for three main solvers, one each for solidification, electromagnetism and fluid flow. Each solver tailored to the respective physics, incorporating a multiscale method that uses varying spacial and temporal scales [16]. The solution 
Table 1: Material properties.

\begin{tabular}{c|l|l||c|l|l}
\hline Variable & Value & Unit & Variable & Value & Unit \\
\hline$c_{p}$ & $1.2 \times 10^{3}$ & $\mathrm{~J} / \mathrm{K} / \mathrm{m}$ & $\sigma$ & $3 \times 10^{6}$ & $\mathrm{~S} / \mathrm{m}$ \\
$L$ & $3.98 \times 10^{5}$ & $\mathrm{~J} / \mathrm{m}^{3}$ & $S_{\mathrm{Al}}$ & $3.5 \times 10^{-6}[17]$ & $\mathrm{V} / \mathrm{K}$ \\
$m$ & 3.5 & $\mathrm{~K} / \%$ & $S_{\mathrm{Cu}}$ & $6.5 \times 10^{-6}[17]$ & $\mathrm{V} / \mathrm{K}$ \\
$k$ & 0.17 & - & $\rho$ & 2860 & $\mathrm{~kg} / \mathrm{m}^{3}$ \\
$D$ & $3 \times 10^{-9}$ & $\mathrm{~m}^{2} / \mathrm{s}$ & $\mu$ & $1.3 \times 10^{-3}$ & $\mathrm{Pas}$
\end{tabular}

procedure essentially has four steps. The first step calculates the transport equations. From the change in local free energy, the second step solves the microstructure evolution using the Enthalpy based method. From the temperature field, phase and composition, the third step solves the thermoelectric currents, providing the driving Lorentz force. Finally, this force is used to solve the fluid flow. The process then repeats with the updated velocities used to calculate transport.

In this work buoyancy is neglected; the alloy under consideration has a relatively low concentration and so density variations in the liquid are small. Furthermore, based on the results presented later, the net buoyancy force can be estimated to be only $1 \%$ of the driving Lorentz force. For higher concentration alloys buoyancy may be an important factor (see [13]). However, the focus here is to independently examine the TEMHD mechanism.

\section{Problem description}

The computational model represents directional solidification of $\mathrm{AlCu}$ $2.5 \%$ wt. in a $6 \mathrm{~K} / \mathrm{mm}$ temperature gradient with a growth rate of $40 \mu \mathrm{m} / \mathrm{s}$. The computational domain is $0.3 \mathrm{~mm}$ thick $(x), 2.4 \mathrm{~mm}$ wide $(y)$ and $1.8 \mathrm{~mm}$ high $(z)$. The domain is meshed with $64 \times 512 \times 394$ cubic cells. The walls of the sample ( $x$ and $y$ domain faces) are assumed to have no flow velocity, be electrically insulating and have no heat or mass flux. The $+\hat{z}$ face represents far field conditions i.e. bulk concentration and zero pressure. Table 1 shows characteristic material property inputs used by the model. The Seebeck power in the solid and liquid is compositionally weighted based on the pure values quoted in Table 1. Furthermore a constant characteristic electrical conductivity is assumed across the entire domain. 


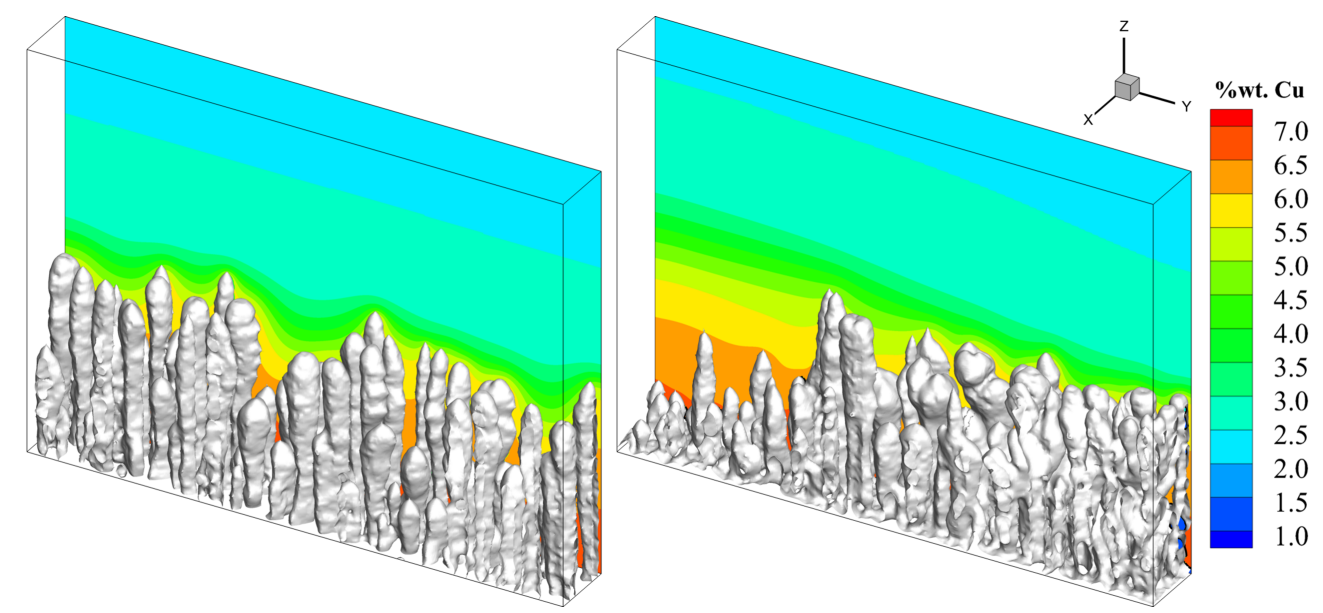

Figure 1: 3-dimensional microstructure and concentration profile. Left: $\mathbf{B}=0$. Right: $\mathbf{B}_{\mathbf{x}}=0.1 \mathrm{~T}$

\section{Results}

Figure 1 shows a comparison of the microstructure and concentration profile after $23 \mathrm{~s}$ of growth for no magnetic field (left) and the application of a $0.1 \mathrm{~T}$ magnetic field (right). In the absence of the magnetic field the numerical results exhibit typical directional solidification behaviour where the interface is flat and the concentration field is relatively constant in the $x, y$ plane. In contrast, the results in the presence of a magnetic field shows a clear difference in microstructure. The interface is no longer flat but convex, with a bias to growth on one side of the domain and stunted growth on the other. Solute concentration is increased in the region of stunted growth representing macrosegregation of the alloy.

A comparison between the computational predictions and experimental results from Wang et al. [4] are given for the interface shape in figure 2 and for the dendrite size and dendrite spacing in figure 3. The experimental results represent an $\mathrm{AlCu} 0.85 \%$ wt. alloy in a $6 \mathrm{~K} / \mathrm{mm}$ temperature gradient with a pull rate of $5 \mu \mathrm{m} / \mathrm{s}$ in a $0.5 \mathrm{~T}$ magnetic field. Although there are significant differences between the simulation parameters and the experiments, such as pull rate, magnetic field intensity and also the model represent a thin sample, while the experiments are cylindrical, there are some very clear similarities between the numerical and experimental results. In both cases the interface in the $y, z$ plane shown in figure 2 forms a convex shape, where 


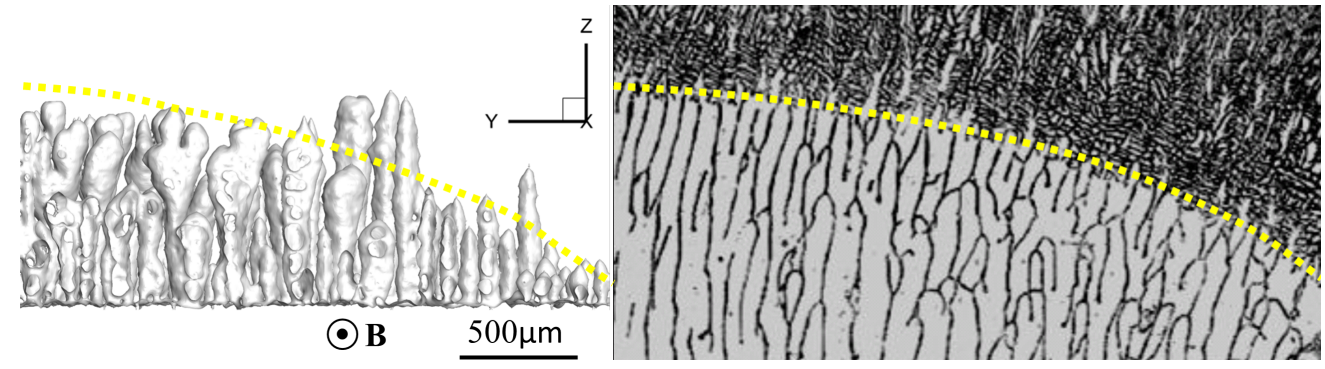

Figure 2: Interface shape. Left: Numerical model. Right: Experimental data from Wang et al. [4]. Note scale bar applies to both results.

the interface is lowest in the region of macrosegregation. Figure 3 shows the microstructure in the $x, y$ plane and how the space between dendrites decreases and the dendrite size increases from the macrosegregation region to the other side of the sample. The similarity between the numerical and experimental results suggests that the underlying mechanism could be the same. To further understand this it is necessary to look at the formation of TEMHD in more detail.

The thermoelectric field $S \nabla T$ is in the direction of the thermal gradient causing current to pass along the inside of the dendrite emanate by the tip and pass through the liquid back down into the mushy zone forming a closed loop. This is highlighted in figure 4 , which shows contours of $\mathbf{J}_{\mathbf{z}}$ in planes parallel and normal to the growth direction. The current is localised to the mushy zone and does not extend into the bulk liquid. In the presence of the magnetic field this current generates a Lorentz force. The solid dendrites are considered to be rigid and the Lorentz force is essentially balanced by structural forces. In the interdendritic liquid the Lorentz force drives fluid flow and the force is balanced by viscous drag, pressure gradient and electromagnetic damping.

As the current is predominantly orientated in $\hat{z}$, the application of a transverse $(\hat{x})$ magnetic field gives rise to a significant force in $\hat{y}$. The current in the interdendritic liquid generates a Lorentz force in $-\hat{y}$, while current emanating from the tips produces a Lorentz force in $+\hat{y}$. The latter can lead to localised flow circulations around the tip [18], but the primary driver for the macrosegregation mechanism comes from the interdendritic forces. To satisfy continuity a large scale flow circulation forms that passes through the interdendritic network, changing direction at the wall and passing back over 


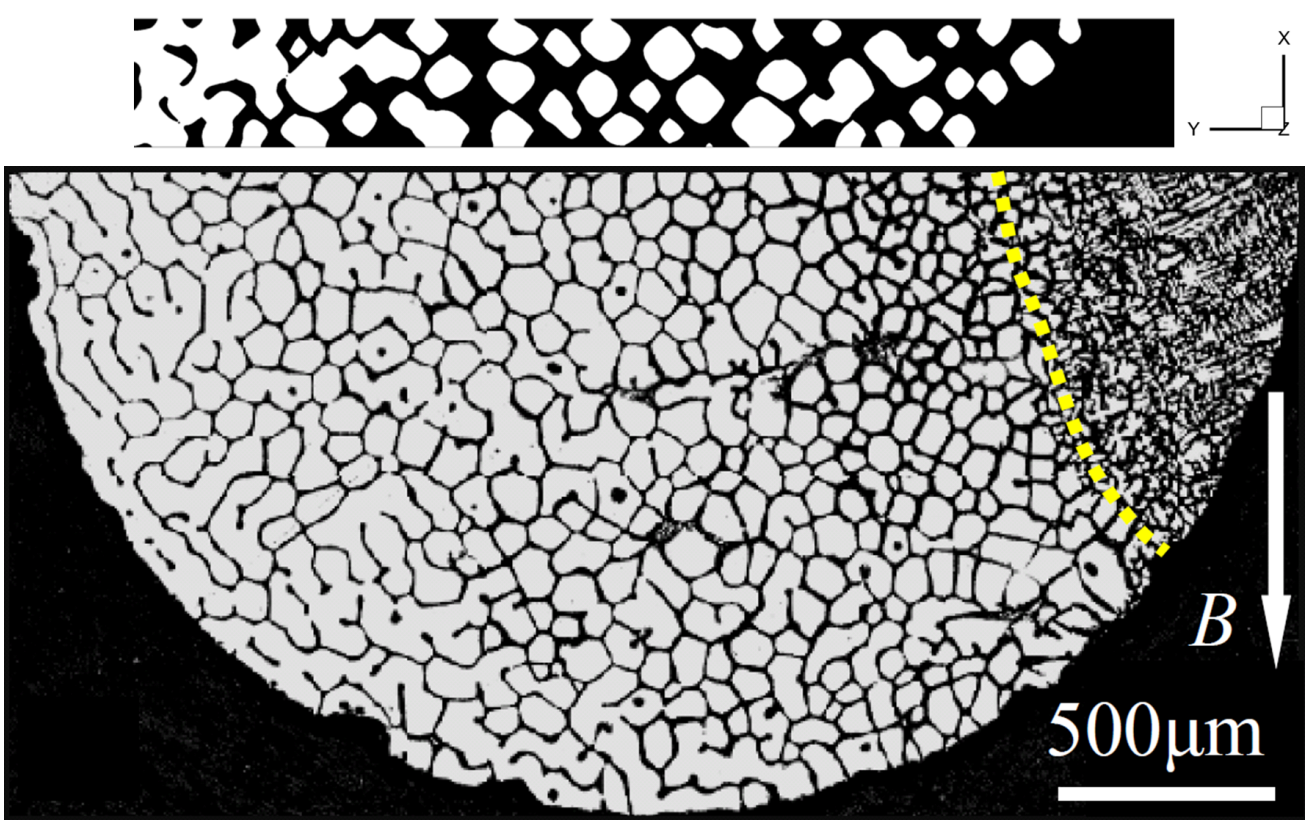

Figure 3: Dendrite size and spacing. Top: Numerical model. Bottom: Experimental data from Wang et al. [4] 


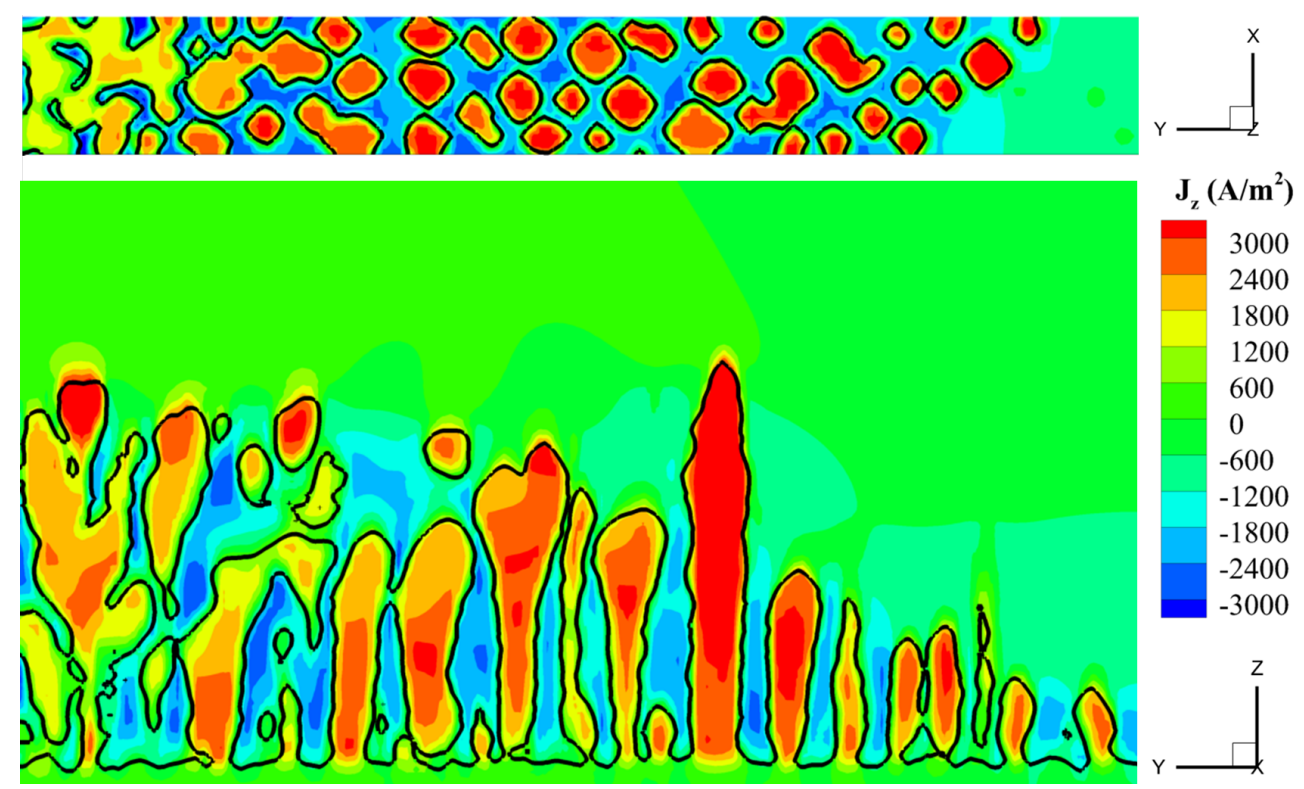

Figure 4: $\mathbf{J}_{\mathbf{z}}$. Top: $x, y$ plane. Bottom: $y, z$ plane

the dendrite tips. All of these flow features can be seen in figure 5, which also highlights the intricate path the flow takes through the interdendritic network.

As the solute concentration of the interdendritic fluid is higher than fluid outside of the dendritic network there is a net transport of solute to one side of the domain. Upward flow near the wall causes this solute to be transported ahead of the solidification front decreasing the the interface velocity in this region. The liquid then flows back across the domain and is incident to the interface. However, as mixing occurs from the bulk, the concentration decreases and the incident flow encourages growth in this region. This incident low concentration flow causes coarsening of the dendrites which both increase dendrite size and also decrease the dendrite spacing. This effect can be seen in both the numerical and experimental results presented in figure 3 .

The numerical model gives an insight into fluid velocity within the interdendritic regions and in the bulk. Under these conditions the model predicts TEMHD velocities $O(200 \mu \mathrm{m} / \mathrm{s})$. Figure 6 shows iso-surfaces of $-100 \mu \mathrm{m} / \mathrm{s}$ and $75 \mu \mathrm{m} / \mathrm{s}$ flow for both $\mathbf{u}_{\mathbf{y}}$ and $\mathbf{u}_{\mathbf{z}}$. The $\mathbf{u}_{\mathbf{y}}$ interdendritic flow is almost entirely in $-\hat{y}$ leading to macrosegregation and the return $\mathbf{u}_{\mathbf{y}}$ flow is in $+\hat{y}$ 


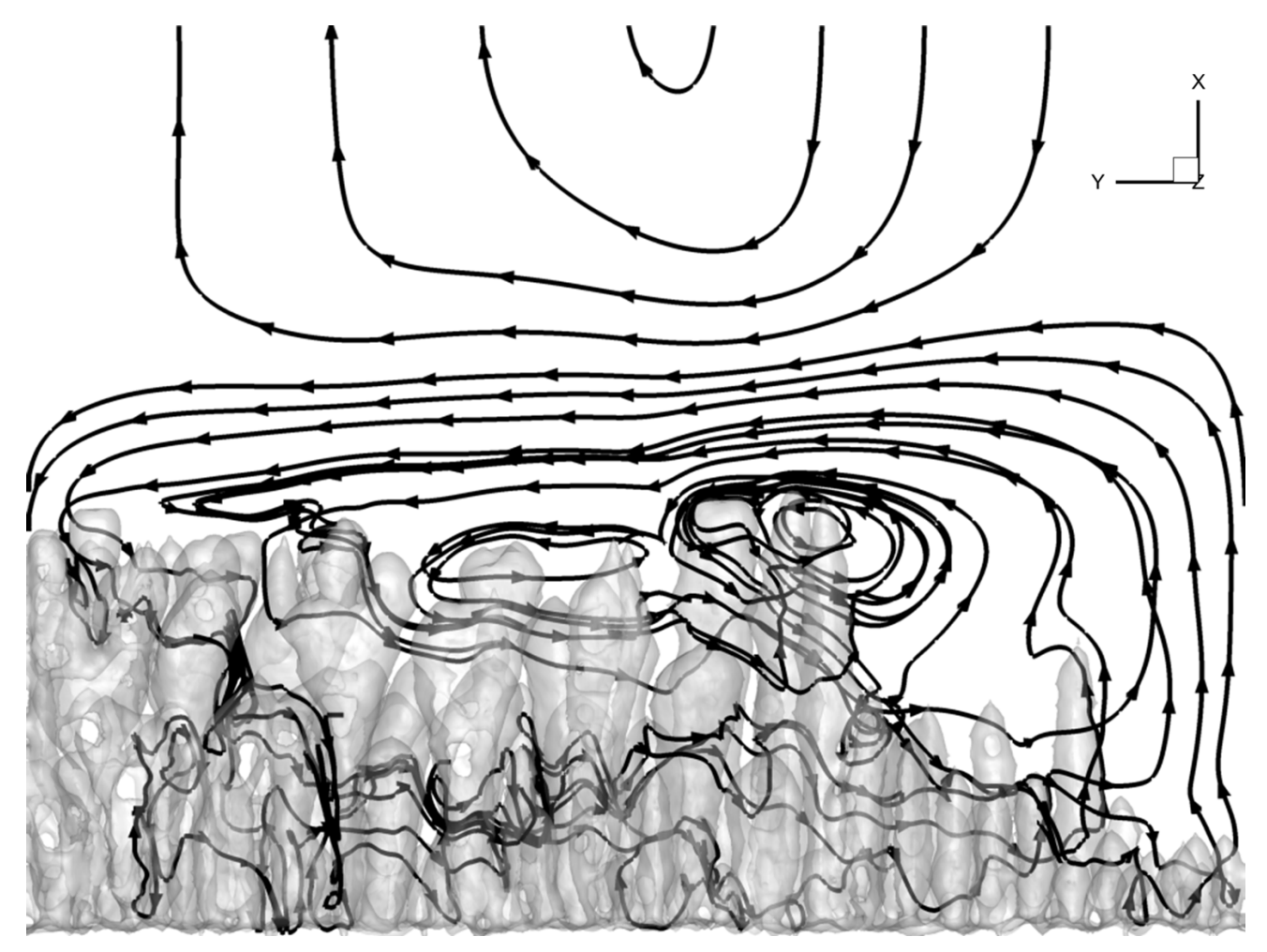

Figure 5: Steamlines of TEMHD 

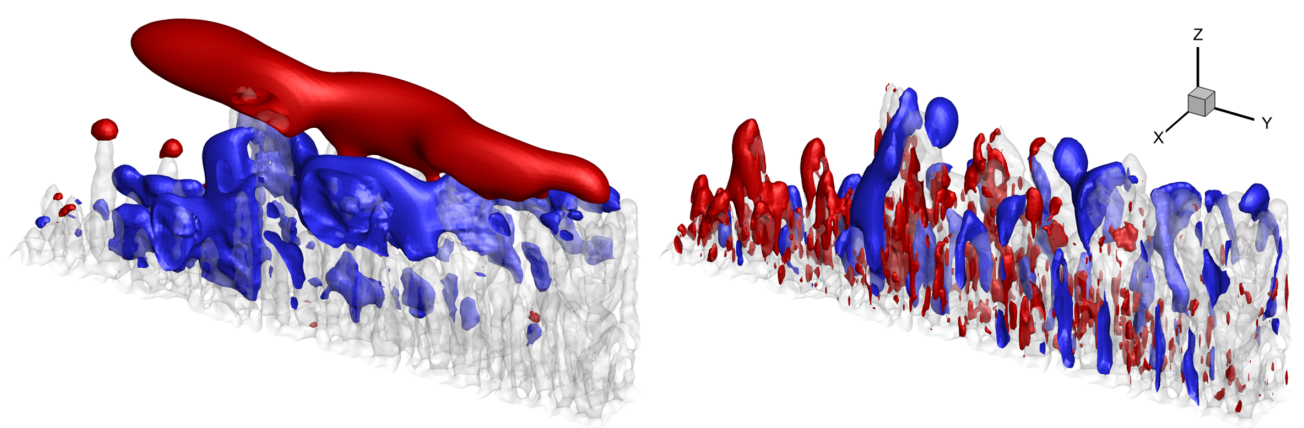

Figure 6: Surfaces of constant velocity. Red: $75 \mu \mathrm{m} / \mathrm{s}$. Blue: $-100 \mu \mathrm{m} / \mathrm{s}$. Left: $\mathbf{u}_{\mathbf{y}}$. Right: $\mathbf{u}_{\mathbf{z}}$.

ahead of the interface. $\mathbf{u}_{\mathbf{z}}$ highlights the complex path flow takes through the mushy zone, where flow enters the mushy zone from the centre of the domain to $+\hat{y}$ and where it leaves in the macrosegregation region. However, the figure also shows that there is very little flow near the lower $+\hat{y}$ edge for both $\mathbf{u}_{\mathbf{y}}$ and $\mathbf{u}_{\mathbf{z}}$ of the domain. In this region coarsening of the dendrites has occurred, the space between dendrites is decreased and the flow is more restricted.

The interdendritic flow is essentially porous media flow, however in traditional porous media flow it is driven by external forces, while in this case the forces are internal to the pores. Consequently the pore size (space between dendrites) may also be a key parameter in this phenomenon. Viscous drag will increase as the spacing between dendrites decreases, but due to the concentration dependency of the thermoelectric field, the current density will also change. This can be seen in the $+\hat{y}$ region of figure 4 , where the increase in volume of solid causes a reduction in current density. However, to preserve continuity of charge, the reduction in volume of neighbouring fluid causes an increase in current density. Hence, current density dependency on localised phase/concentration will modify the driving Lorentz force in the liquid.

In this work the dendrites on the $+\hat{y}$ side of the sample become coarser with the application of a $0.1 \mathrm{~T}$ magnetic field compared to no magnetic field. While this agrees well with the presented experimental results, other experiments with similar experimental conditions [6] have also observed a refinement of the dendrites in this region. These differing findings indicate that there may be other mechanisms that become dominant under different con- 
ditions. Further investigations into parameters such as pull rate, magnetic field intensity and sample geometry (i.e. cylindrical) are necessary to fully understand this phenomenon.

\section{Conclusion}

The application of a transverse magnetic field has been shown both computationally and experimentally to modify the microstructural evolution of alloys during directional solidification. The numerical results of this work have been compared to a specific experimental example and shown changes similar to those observed. The primary driver for this mechanism has been identified as the strong Lorentz force formed in the interdendritic region due to the interaction between thermoelectric currents and the externally applied transverse magnetic field. This leads to several significant changes, including macrosegregation, dendrite size and the spacing between dendrites. The numerical modelling has given insight into the magnitude of TEMHD velocities within the interdendritic region, which experimentally, is a very difficult feat to achieve.

The ability to introduce contactless forces deep within the mushy zone is of considerable interest both scientifically and industrially. This phenomenon, if fully understood, has the potential to provide an additional control mechanism to the microstructure formation through tailored design of the magnetic field.

\section{Acknowledgements}

The authors would like to acknowledge the UK's Engineering and Physical Sciences Research Council (EPSRC) (EP/K011413/1, EP/K007734/1) for funding and support provided by the Research Complex at Harwell, funded in part by the EPSRC (EP/I02249X/1). A representative sample of research data is provided in supplementary data at gala.gre.ac.uk. The underlying raw data is not shared online due to its large size.

[1] R. Moreau, O. Laskar, M. Tanaka, D. Camel, Thermoelectric magnetohydrodynamic effects on solidification of metallic alloys in the dendritic regime, Materials Science and Engineering: A 173, Issues 1-2 (1993) 93-100. 
[2] R. Moreau, O. Laskar, M. Tanaka, Thermoelectric and magnetohydrodynamic effects on solidifying alloys, Magnetohydrodynamics 32 (1996) 173-177.

[3] P. Lehmann, R. Moreau, D. Camel, R. Bolcat, Modification of interdendritic convection in directional solidifcation by a uniform magnetic field, Acta Materialia 46, No. 11 (1998) 4067-4079.

[4] J. Wang, Z. Ren, Y. Fautrelle, X. Li, H. Nguyen-Thi, N. MangelinckNoel, G. S. A. Jaoude, Y. Zhong, I. Kaldre, A. Bojarevics, Modification of liquid/solid interface shape in directionally solidifying alcu alloys by a transverse magnetic field, Journal of Materials Science 48-1 (2013) 213-219.

[5] I. Kaldre, Y. Fautrelle, J. Etay, A. Bojarevics, L. Buligins, Thermoelectric current and magnetic field interaction influence on the structure of directionally solidified sn10 wt.\% pb alloy, Journal of Alloys and Compounds 571 (2013) 50-55.

[6] X. Li, Z. Ren, A. Gagnoud, O. Budebkova, Y. Fautrelle, Effects of thermoelectric magnetic convection on the solidification structure during directional solidification under lower transverse magnetic field, Metallurgical and Materials Transactions A 42-11 (2011) 3459-3471.

[7] X. Li, A. Gagnoud, Z. Ren, Y. Fautrelle, R. Moreau, Investigation of thermoelectric magnetic convection and its effect on solidification structure during directional solidification under a low axial magnetic field, Acta Materialia 57 (2009) 2180-2197.

[8] X. Li, A. Gagnoud, Y. Fautrelle, Z. Ren, R. Moreau, Influence of thermoelectric effects on the morphology of alsi eutectic during directional solidification under an axial strong magnetic field, Journal of Crystal Growth 367 (2013) 94-103.

[9] X. Li, Y. Fautrelle, K. Zaidat, A. Gagnoud, Z. Ren, R. Moreau, Y. Zhang, C. Esling, Columnar-to-equiaxed transitions in al-based alloys during directional solidification under a high magnetic field, Journal of Crystal Growth 312 (2010) 267-272.

[10] X. Li, Y. Fautrelle, Z. Ren, Influence of thermoelectric effects on the solid-liquid interface shape and cellular morphology in the mushy zone 
during the directional solidification of al-cu alloys under a magnetic field, Acta Materialia 55 (2007) 3803-3813.

[11] H. Yasuda, K. Nogita, C. Gourlay, M. Yoshiya, T. Nagira, In-situ observation of sn alloy solidification at spring8, Journal of the Japan Welding Society 78 (2009) 6-9.

[12] J. A. Shercliff, Thermoelectric magnetohydrodynamics, Journal of Fluid Mechanics 91, part 2 (1978) 231-251.

[13] A. Kao, N. Shevchenko, O. Roshupinka, S. Eckert, K. Pericleous, The effects of natural, forced and thermoelectric magnetohydrodynamic convection during the solidification of thin sample alloys, IOP Conf. Series: Materials Science and Engineering 84 (2015) 012018.

[14] A. Kao, K. Pericleous, A numerical model coupling thermoelectricity, magnetohydrodynamics and dendritic growth, Journal of Algorithms and Computational Technology 6 No. 1 (2012) 173-201.

[15] V. R. Voller, An enthalpy method for modeling dendritic growth in a binary alloy, International Journal of Heat and Mass Transfer 51 (2008) 823-834.

[16] A. Kao, K. Pericleous, A multi scale method for thermoelectric mhd in dendritic solidification, Journal of Iron and Steel International 19 (S1-1) (2012) 317-321.

[17] C. J. M. Lasance, The seebeck coefficient, Electronics Cooling Magazine 12, No. 4.

[18] A. Kao, Analytic solutions to determine critical magnetic fields for thermoelectric magnetohydrodynamics in alloy solidification, Metallurgical and Materials Transactions A 46 (9) (2015) 4215-4233. 